\title{
Channel Estimation Algorithm for mmWave Communication with Array Antennas via Reduced-Dimension MUSIC
}

\author{
Wei-Tao LIU1,a, Zhong-Xi XIA ${ }^{2, b}$, Qian-Lin CHENG ${ }^{3, c}$, Dong-Lin YANG ${ }^{4, d}$, \\ Xiao-Fei ZHANG ${ }^{5, \mathrm{e}}$
}

${ }^{1}$ Nanjing University of Aeronautics and Astronautics

Nanjing, China

liuweitao@nuaa.edu.cn xiazhongxi@nuaa.edu.cn chengqianlin nuaa@163.com yangdl 2012@163.com zhangxiaofei@nuaa.edu.cn

\begin{abstract}
Keywords: Channel estimation, Array antennas, mmWave communication.
Abstract: This paper considers channel estimation for mmWave communication with massive received array antennas at $\mathrm{BS}$. Instead of estimate the channel matrix, we resolve the problem into two steps. Firstly, one could separately estimate DOA, on the basis of the array signal processing techniques, and then estimate the corresponding path attenuation according to the pilots from the transmitting terminals. This method is analyzed and compared with conventional LS channel estimation algorithm. The performance of the suggested algorithm precedes LS algorithm without large number of pilots. Finally, numerical simulation results are provided to verify the effectiveness of our channel estimation approach.
\end{abstract}

\section{Introduction}

In consideration of the crowded spectrum, people have put more and more attention to the higher band surrounding $60 \mathrm{GHz}$, which was allocated internationally in former years. The advantage of the short wavelength of mmWave can be fully utilized to expand the scale of antenna arrays [1]. Hence, the base station (BS) will make it possible to integrate dozens of hundreds of antennas in a small area [2]. As a result, the communication capability, including the accuracy of direction of arrival (DOA), the beamforming gain and so on, obtains huge improvement under the condition of massive antenna arrays, which has been studied extensively[3][4][5]. At the same time of noticing the advantage of mmWave, people don't neglect the restrictions of hardware circuit resources. Traditionally, every antenna cell connects to a strip of RF chains, which occupies most of the system. Hence, it's inadvisable to magnify blindly the number of antenna at BS.

There is an important shortage of mmWave that the atmosphere and the rain could impose great path loss during outdoor propagation process. It's important for the BS to gain the more detailed channel state information (CSI) the better. At the same time, resulting from the huge number of antenna array, saving resources used on the channel estimation becomes significant. In OFDM system, a small number of training sequences are used to obtain the CSI, which greatly decrease the complexity before transferring messages. In [6], LS (Least Square) and MMSE (Minimum Mean Square Error) algorithm are employed by additive pilots before useful signals. This method is rather simple, but at the price of reducing the accuracy. Two-dimensional multiple signal classification (2D-MUSIC) algorithm is considered when estimating DOAs. Precise azimuth and elevation angles are obtained from the signals transmitted by terminals to the BS. However, 2D-MUSIC algorithm needs a peak-search procedure, which has too high complexity to apply to the actual.

Because the wireless channel at $60 \mathrm{GHz}$ has high attenuation due to oxygen absorption [7], beamforming techniques are necessary for the new generation wireless communication. Hence, DOAs are necessary not for the channel estimation but the effectiveness of beamforming. At present, not a few researchers have done abundant work on DOA algorithms. The methods of two-dimensional DOA estimation includes the 2D-MUSIC algorithm [8], 2D-ESPRIT algorithm [9], the propagator method (PM) algorithm [10] and so on. 
The rest of this paper is organized as follows. In section II, we build up a 60GHz system model and analyze the data model of this system. Then we discuss the process of the channel estimation, which focuses on path attenuation DOA algorithms in section III. In section IV, the MATLAB simulation results are presented. Finally, we draw a conclusion in section V.

\section{Data Model}

This paper considers the scenario that the receiver is aligned by $N_{r}=M \times N$ antennas, and every user owns signal antenna. We just considers the uplink that $\mathrm{K}$ users transmit signals to the BS. As Fig.1 shows, we assume that the signal impinges on the antenna array directly. The users are located in the direction (elevation angle $\theta$ and azimuth angle $\phi$ ) from the BS.

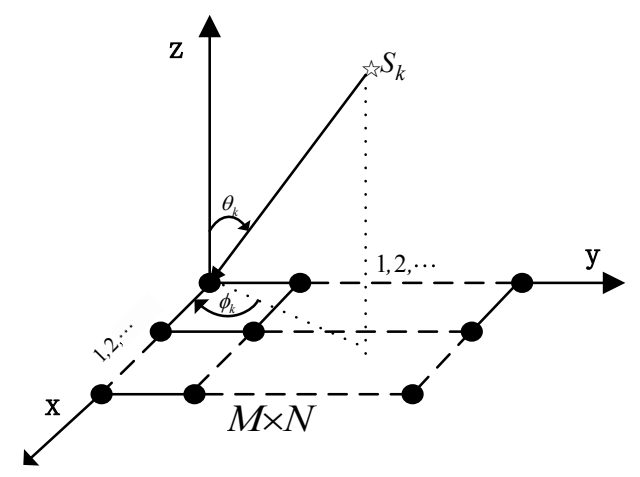

Fig.1 Planar array geometry

The received signals, as in $[11,12]$, could be expressed as follows

$$
Y=H S+N
$$

where $S=\left[s_{1}^{T}, s_{2}^{T}, \ldots, s_{K}^{T}\right]^{T}, s \in \square$ is the signal vector sent by every user. $L$ is the number of snapshots. $N$ is a $N_{r} \times L$ matrix, whose elements are uncorrelated zero mean circularly symmetric complex Gaussian noise. $Y$ is the received matrix whose dimension is also $N_{r} \times L$. $H$ means the channel matrix. This paper expresses it as

$$
H=A(\theta, \phi) \square,
$$

where $A(\theta, \phi)$ is the directional matrix of the antenna array, and $\theta \in[0, \pi / 2), \phi \in[0,2 \pi)$. $\Lambda=\operatorname{diag}\left(\lambda_{1}, \lambda_{2}, \ldots \quad \ldots \quad\right.$ represents the path attenuation, and the diagonal elements $\lambda_{k} \sim C N(0,1)(k=1,2 \cdots \quad$ is the complex gain of the k-th propagation path. According to Eq.2, we estimate DOA and $\Lambda$ instead of channel matrix directly.

\section{Channel Estimation}

In this section, we analyze the channel estimation algorithms. In the tradition, LS algorithm and MMSE algorithm are used usually to estimate flat fading MIMO channel with the help of training sequences. Even though the LS algorithm is simple, it perform not very well at the low signal-to-noise-ratio (SNR). And the MMSE algorithm promotes its performance by applying the signal and noise information, which also resulting in the complexity enhancement. This paper estimates DOA firstly and then use the achieved directional matrix to obtain path attenuation values. Finally, the channel matrix is restructured by collecting the estimated parameters above.

\section{Directional Matrix}


Before elaborating the DOA estimation algorithm, the antenna array structure and its characteristic are important for our deeper research. As Fig. 1 shows, the received antenna arrays consists of $M \times N$ elements, which are uniformly distributed with spacing distance $d$ between two random neighboring elements in a small area. Suppose that a spatial narrowband plane wave with the wavelength of $\lambda$, and with the angle $\left(\theta_{k}, \phi_{k}\right)(k=1,2 \cdots \quad$ entering the antenna model.

The directional vectors on the x-axis and y-axis: $\mathbf{a}_{x}\left(\theta_{k}, \phi_{k}\right)=\left[\begin{array}{lllll}1 & e^{j 2 \pi d \sin \theta_{k} \cos \phi_{k} / \lambda} & \ldots & { }^{-{ }^{\prime}}{ }^{1-1) d s \sin \theta_{k} \cos \phi_{k} / \lambda}\end{array}\right]^{T}$, $\mathbf{a}_{y}\left(\theta_{k}, \phi_{k}\right)=\left[\begin{array}{lllll}1 & e^{j 2 \pi d \sin \theta_{k} \sin \phi_{k} / \lambda} & \ldots & \cdots & \cdots \cdot-1) d \sin \theta_{k} \sin \phi_{k} / \lambda\end{array}\right]^{T}$.

The directional matrix on the $\mathrm{x}$-axis and $\mathrm{y}$-axis can be written as

$$
\begin{aligned}
& \mathrm{A}_{x}=\left[\begin{array}{llll}
\mathbf{a}_{x}\left(\theta_{1}, \phi_{1}\right) & \mathbf{a}_{x}\left(\theta_{2}, \phi_{2}\right) & \ldots & \ldots
\end{array}\right. \\
& \mathrm{A}_{y}=\left[\begin{array}{llll}
\mathbf{a}_{y}\left(\theta_{1}, \phi_{1}\right) & \mathbf{a}_{y}\left(\theta_{2}, \phi_{2}\right) & \ldots & \ldots
\end{array}\right.
\end{aligned}
$$

The antenna array directional matrix can be expressed as

$$
A=\left[\begin{array}{c}
A_{x} D_{1}\left(A_{y}\right) \\
A_{x} D_{2}\left(A_{y}\right) \\
\vdots \\
A_{x} D_{M}\left(A_{y}\right)
\end{array}\right]_{\in \sqcap \cdots \cdots},
$$

where $D_{m}(\square$ is a diagonal matrix structured by the n-th row of the matrix in the parentheses.

\section{DOA Estimation}

Reduced-Dimension MUSIC (RD-MUSIC) algorithm has much lower complexity than 2D-MUSIC, and is capable of reaching very high precision than other algorithms in DOA estimation.

We assume that some basic knowledge about 2D-MUSIC has been mastered and obtain easily the space spectrum function of 2D-MUSIC, expressed as this:

$$
f_{2 D-M U S I C}=\frac{1}{\left[\mathrm{a}_{y}(\theta, \phi) \otimes \mathrm{a}_{x}(\theta, \phi)\right]^{H} E_{n} E_{n}^{H}\left[\mathrm{a}_{y}(\theta, \phi) \otimes \mathrm{a}_{x}(\theta, \phi)\right]},
$$

where $E_{n}$ is the noise subspace, and $\otimes$ means Kronecker product. We define $\mathrm{u} \square$

$v \square \quad: \phi$. In the condition of without noise, the signal subspace could be expressed as

$$
E_{s}=\left[\begin{array}{lllll}
A_{x} & A_{x} \Phi & \cdots & { }^{-T}-1
\end{array}\right]^{T} T,
$$

where $\mathrm{T}$ is a $K \times K$ full rank matrix. Decompose $E_{s}$ into $E_{s}=\left[E_{s 1}{ }^{T}, E_{s 2}{ }^{T}, \cdots \cdots E_{s n} \in \square^{\cdots} \quad \cdots \quad\right.$. Then, we can get

$$
E_{s 1}^{+} E_{s 2}=T^{-1} \Phi_{y} T
$$

Assume that $\beta_{k}$ is the $k$-th eigenvalue of $E_{s 1}{ }^{+} E_{s 2}$, and the diagonal element of $\Phi_{y}$. Therefore, the initial estimated value of $\sin \alpha_{k} \sin \theta_{k}$ is $u_{k}^{i n i}=-\operatorname{angle}\left(\beta_{k}\right) \lambda /(2 \pi d)$, where angle( $\square$ is the phase of a complex number.

We define 


$$
\begin{aligned}
& V(u, v)=\left[\mathrm{a}_{y}(u) \otimes \mathrm{a}_{x}(v)\right]^{H} E_{n} E_{n}{ }^{H}\left[\mathrm{a}_{y}(u) \otimes \mathrm{a}_{x}(v)\right] \\
& =\mathrm{a}_{x}(v)\left[\mathrm{a}_{y}(u) \otimes I_{M}\right]^{H} E_{n} E_{n}{ }^{H}\left[\mathrm{a}_{y}(u) \otimes I_{N}\right] \mathrm{a}_{x}(v), \\
& =\mathrm{a}_{x}(v) Q(u) \mathrm{a}_{x}(v)
\end{aligned}
$$

where $Q(u)=\left[\mathrm{a}_{y}(u) \otimes I_{M}\right]^{H} E_{n} E_{n}{ }^{H}\left[\mathrm{a}_{y}(u) \otimes I_{N}\right]$. To eliminate the trivial solution of $\mathrm{a}_{x}(v)=0_{M}$, we consider make use of $e_{1}^{H} \mathrm{a}_{x}(v)=1, e_{1}=\left[1,0, \cdots, \quad \square^{\cdots}\right.$. Write it as

$$
\min _{u, v}=\mathrm{a}_{x}(v)^{H} Q(u) \mathrm{a}_{x}(v) \text {, s.t. } e_{1}^{H} \mathrm{a}_{x}(v)=1 .
$$

By building up cost function and dealing with the partial derivatives with respect to $a_{x}(v)$. Then we can get

$$
\hat{\mathrm{a}_{x}}(v)=\frac{Q(u)^{-1} e_{1}}{e_{1}^{H} Q(u)^{-1} e_{1}}
$$

Substitute $\hat{a_{x}}(v)$ into Eq.10, we get $\hat{u_{k}}=\arg \max _{u} e_{1}^{H} Q(u)^{-1} e_{1}, k=1,2, \cdots \quad$.Search $u \in\left[u_{k}^{\hat{i n i}}-\square\right.$ ( $\square \quad$ is a small value) to find the maximum values of all the $(1,1)$-th elements of $Q(u)^{-1}$. The $K$ peaks are the estimated values $\left(\hat{u}_{1}, \hat{u}_{2}, \cdots^{\wedge}\right.$. According to (14), we have obtained $\hat{\mathrm{a}}_{x}\left(v_{1}\right), \hat{\mathrm{a}}_{x}\left(v_{2}\right), \ldots{ }^{\wedge}$. Because $\mathrm{a}_{x}\left(v_{k}\right)=\left[\begin{array}{lllll}1 & e^{j 2 \pi v_{k} / \lambda} & \cdots & \cdots & 1-1) d v_{k} / \lambda\end{array}\right]^{T}$,

$$
\begin{aligned}
& \mathrm{g}_{\mathrm{k}}=\operatorname{angle}\left(\mathrm{a}_{x}\left(v_{k}\right)\right) \\
& \left.=\left[0,2 \pi d v_{k} / \lambda, \cdots \quad 1-1\right) v_{k} / \lambda\right]^{T}=v_{k} \mathrm{q},
\end{aligned}
$$

where $\mathrm{q}=[0,2 \pi d / \lambda, \cdots \quad 1-1) / \lambda]^{T}$. Make use of the least square rule to estimate $v_{k}$, we can get $\min _{c_{k}}\left\|\mathrm{Pc}_{k}-\hat{\mathrm{g}}_{k}\right\|_{F}^{2}$.where $\hat{\mathrm{g}}_{k}=\operatorname{angle}\left(\hat{\mathrm{a}}_{x}\left(v_{k}\right)\right), \mathrm{c}_{k}=\left[\mathrm{c}_{k 0}, \mathrm{c}_{k 1}\right]^{T} \in \square^{-}, \mathrm{c}_{k 1}$ is the estimated value of $v_{k}$.

$$
\begin{gathered}
\mathrm{P}=\left[\begin{array}{cc}
1 & 0 \\
1 & 2 \pi d / \lambda \\
\vdots & \vdots \\
1 & 2 \pi d(M-1) / \lambda
\end{array}\right] \\
\left.\hat{\mathrm{c}}_{k 0}, \hat{v}_{k}\right]^{T}=\left(\mathrm{P}^{T} \mathrm{P}\right)^{-1} \mathrm{P}^{T} \hat{\mathrm{g}}_{k} .
\end{gathered}
$$

So we can get $\hat{v_{k}}$. According to the analysis above, we have estimated $\hat{u_{k}}$ and $\hat{v_{k}}$, therefore, $\hat{\theta}_{k}=\sin ^{-1}\left(\operatorname{abs}\left(\hat{v_{k}}+j \hat{u_{k}}\right), \hat{\phi}_{k}=\operatorname{angle}\left(\hat{v_{k}}+j \hat{u_{k}}\right)\right.$.

Obviously, RD-MUSIC needn't peak search as MUSIC, so that the complexity reduces by a large margin. The complexity of RD-MUSIC is $O\left\{n_{l} K\left[\left(M^{2} N+M^{2}\right)(M N-K)+M^{2}\right]+L M^{2} N^{2}+M^{3} N^{3}+2 K^{2} M+3 K^{3}\right\}$. And the MUSIC's complexity reaches $O\left\{L M^{2} N^{2}+M^{3} N^{3}+n_{g}[M N(M N-K)+M N-K]\right\}$. $n_{l}$ and $n_{g}$ represent the time of partial and global search.

\section{Path attenuation}

For estimating the attenuation, some pilots are used and known to the BS. We replace the signal source with the pilot, expressed as $P$. The length of pilots is $l_{P}$ Also, through the DOA estimation above, the BS is able to achieve A. According to Eq.1, we can calculate that 


$$
\begin{aligned}
& A^{+} Y P^{+}=A^{+}(A \Lambda P+N) P^{+} \\
& =A^{+} A \Lambda P P^{+}+A^{+} N P^{+}
\end{aligned}
$$

If ignore the affect of noise, we can get $\Lambda \approx A^{+} Y P^{+}$.At last, $\left[\hat{\lambda}_{1}, \hat{\lambda}_{2} \ldots \hat{\lambda}_{K}\right]=\operatorname{diag}(\Lambda), \hat{\Lambda}=\operatorname{diag}\left(\hat{\lambda}_{1}, \hat{\lambda}_{2}, \ldots{ }^{\wedge} \quad\right.$.

It's necessary to declare that the length of pilots must be longer than the number of users to meet the requirement that $\mathrm{P}$ has full row rank. Hence, the channel matrix estimation can be expressed as

$$
\hat{H}=A\left(\hat{\theta}_{k}, \hat{\phi}_{k}\right){ }^{\wedge} \text {. }
$$

The analysis above has proved that the solving process of the channel matrix. The detailed algorithm steps are summarized as follows:

Step 1: Conduct eigenvalues decomposition of the signal's correlation matrix, get $E_{s}$ and $E_{n}$.

Step 2: Initial estimation according to $E_{s}$. Find the maxium $K$ peaks from the $(1,1)$-th elements of $Q(u)^{-1}$ by patial search, then get corresponding estimated $u_{k}(1, \cdots$

Step 3: According to Eq.11, get $\hat{\mathrm{a}}_{x}\left(v_{k}\right)$, and then obtain $\hat{v}_{k}$ using method of Least Squares. Then estimate 2D-DOA according to equation above and substitute the achieved angular information $\left(\hat{\theta}_{k}, \hat{\phi}_{k}\right)$ into Eq.5, namely get estimated $A\left(\hat{\theta}_{k}, \hat{\phi}_{k}\right)$.

Step 4: Get path attenuation coefficients $\hat{\lambda}_{k}$. Combine the twofold estimation above to achieve channel matrix.

The computional complexity of traditional LS channel estimation algorithm is $O\left\{l_{P} K M N+2 K l_{P}{ }^{2}+l_{P}{ }^{3}\right\}$, and our proposed method's is $O\left\{l_{P} K M N+2 K l_{P}{ }^{2}+l_{P}{ }^{3}+2 K^{2} M N+K^{3}+K^{2} l_{P}+K M N\right\}$.

Because the LS algorithm is based on the pseudo-inverse of the pilot, the complexity is simpler than our method. As a result, the performance of LS is a little lower that will be shown in simulation results.

\section{Simulation Results}

Before the simulation, we assume that the transmitter and the receiver are stationary. There are three $(\mathrm{K}=3)$ transmitters and each one has just one antenna, and the receiver antenna array have $8 \times 8$ whose spacing distance is half wavelength, that is $d=\lambda / 2$. Eq.17 represents the root-mean-square error (RMSE) of DOA estimation.

$$
R M S E=\frac{1}{K} \sum_{k=1}^{K} \sqrt{\frac{1}{Q} \sum_{n=1}^{Q}\left[\left(\hat{\alpha}_{k, n}-\alpha\right)^{2}+\left(\hat{\theta}_{k, n}-\theta\right)^{2}\right]},
$$

where $\hat{\alpha}_{k, n}$ and $\hat{\theta}_{k, n}$ are the $\mathrm{k}$-th target's estimated values of $\mathrm{n}$-th Monte-Carlo simulation. $\mathrm{Q}$ is the times of Monte Carlo trail. the gross simulation time is 1000 . We assume that the elevation and azimuth angles are $\left(10^{\circ}, 10^{\circ}\right),\left(20^{\circ}, 20^{\circ}\right)$, and $\left(30^{\circ}, 30^{\circ}\right)$. Fig. 2 shows that RD-MUSIC algorithm and MUSIC algorithm are able to estimate more and more accurate angles with the increase of SNR and the former has adjacent performance to MUSIC algorithm which has much higher complexity and expand more computational cost than our algorithm.

When estimating the attenuation by pilots, mean square error (MSE) is used to measure the channel estimation and express it as

$$
M S E=\frac{1}{N_{r} \square}\|H-\hat{H}\|_{F}^{2},
$$


where $\|[]_{n}$ means Frobenius norm of a matrix or vector.

Fig. 3 indicates MSE of these methods with the increase of SNR. At low SNR, because RD-MUSIC algorithm is more accurate in DOA estimation, it outperforms other algorithms including LS algorithm. It's obvious that the channel estimation which includes DOA estimation has lower MSE than traditional LS algorithm. And at low SNR, our proposed algorithm also keeps very low MSE compared with others. We also find that channel estimation including DOA except PM algorithm has similar MSE performance because of the inaccuracy of path attenuation estimation.

The length of pilots has an influence of the MSE performance. Here, Hadamard matrix is used to produce pilot sequences. The lengths of pilots on x-axis are 4, 8, 16, 32 and 64. As Fig.4 shows, the accuracy becomes better as the increase of the length of pilots, which should be bigger than K. However, the MSE curve keep constant gradually. Moreover, the proposed method has lower MSE than LS algorithm and pretty similar performance to MUSIC algorithm.

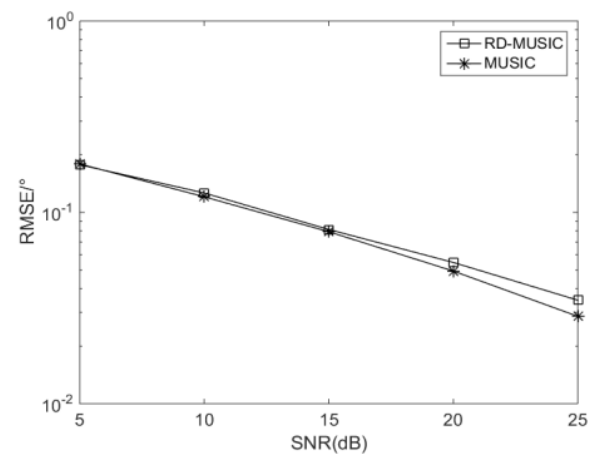

Fig.2 RMSE comparison

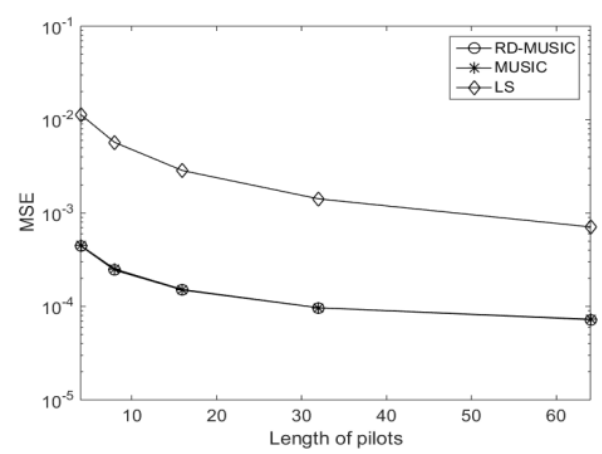

Fig.4 MSE for different length of pilots

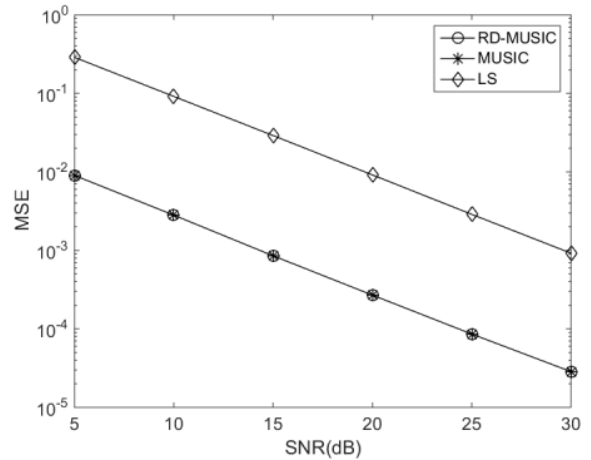

Fig.3 MSE of the channel estimation algorithms

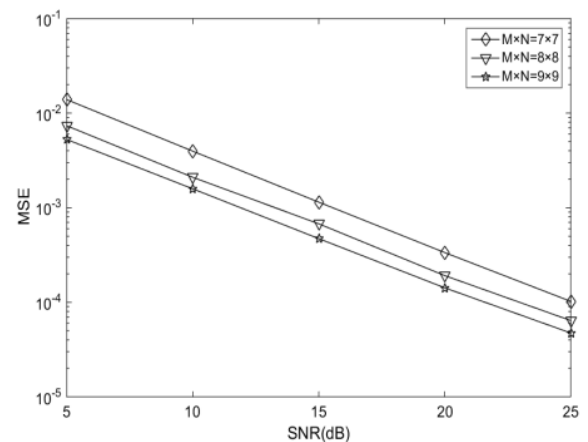

Fig. 5 MSE for different numbers

When the number of users is constant, the increasement of the received antennas causes more accurate on DOA estimation and then get lower MSE. As Fig. 5 conveys to us, the quadrate distributions of the antenna array are set to $7 \times 7,8 \times 8$ and $9 \times 9$. In practice, the huge number of antennas owes to the $60 \mathrm{GHz}$ system, which has come true in some aspects and will have more comprehensive in the future.

\section{Summary}

The paper presents a novel channel estimation algorithm which contains DOA estimation and path attenuation. Different from traditional channel estimation algorithm, this method make full use of source signals to estimate DOA information and pilots to estimate attenuation coefficients. The advantage mainly lies in that on the one hand it obtains more precise MSE curve, and on the other hand saves transmitting resources for channel estimation. As the simulation results show, the algorithm we propose outperforms traditional LS algorithm and has lower complexity than 2D-MUSIC algorithm in DOA estimation. Moreover, in path attenuation estimation, the length of pilots are critical to be considered when the data size is restricted on the transmitter sides. 


\section{Acknowledgement}

This work is supported by China NSF Grants $(61371169,61601167)$, the open research fund of National Mobile Communications Research Laboratory, Southeast University (No.2015D030), Jiangsu NSF (BK20161489), the open research fund of State Key Laboratory of Millimeter Waves, Southeast University (No. K201826), and the Fundamental Research Funds for the Central Universities(NO: NE2017103).

\section{References}

[1] S. Hur, T. Kim, D. Love, J. Krogmeier, T. Thomas, and A. Ghosh, "Millimeter wave beamforming for wireless backhaul and access in small cell networks," IEEE Trans. Commun., vol. 61, no. 10, pp. 4391-4403, Oct. 2013.

[2] W. Tabikh, D. Slock and Y. Yuan-Wu, "Optimal beamforming with combined channel and path CSIT for multi-cell multi-user MIMO,” 2016 Information Theory and Applications Workshop (ITA), La Jolla, CA, 2016, pp. 1-6.

[3] W. Roh et al., "Millimeter-wave beamforming as an enabling technology for 5G cellular communications: theoretical feasibility and prototype results," IEEE Communications Magazine, vol. 52, no. 2, pp. 106-113, February 2014.

[4] P. Liu, M. Di Renzo and A. Springer, "Line-of-Sight Spatial Modulation for Indoor mmWave Communication at $60 \mathrm{GHz}$," IEEE Transactions on Wireless Communications, vol. 15, no. 11, pp. 7373-7389, Nov. 2016.

[5] A. Rozé, M. Crussière, M. Hélard and C. Langlais, "Comparison between a hybrid digital and analog beamforming system and a fully digital Massive MIMO system with adaptive beamsteering receivers in millimeter-Wave transmissions," 2016 International Symposium on Wireless Communication Systems (ISWCS), Poznan, 2016, pp. 86-91.

[6] J. I. Nadar, J. Koti and D. Jayaswal, “OFDM pilot based channel estimation,” 2014 International Conference on Advances in Engineering \& Technology Research (ICAETR - 2014), Unnao, 2014, pp. $1-5$.

[7] T. Zarrouk, A. El Moussati, M. El Yakyaoui and A. El Oualkadi, "On 60GHz wireless link performance in indoor and outdoor environments based on IEEE 802.15.3c," 2015 4th International Conference on Electrical Engineering (ICEE), Boumerdes, 2015, pp. 1-5.

[8] Wax M, Shan T J, Kailath T. "Spatio-temporal spectral analysis by eigenstructure methods," IEEE Transactions on Acoustics Speech \& Signal Processing, 1984, 32(4):817-827.

[9] Zoltowski M D, Haardt M, and Mathews C P. "Closed-form 2-D angle estimation with rectangular arrays in element space or beamspace via unitary ESPRIT," Signal Processing IEEE Transactions on, 1996, 44(2):316-328.

[10] Tayem N and Kwon H M. "L-shape 2-dimensional arrival angle estimation with propagator method," IEEE Transactions on Antennas \& Propagation, 2005, 53(5):1622-1630.

[11]Van d B J. "On channel estimation in OFDM systems," Proc.ieee Vehicular Technology Conf.chicago IL, 1995, 2(2):815-819 vol.2.

[12] Fan D, Zhong Z and Wang G, "Channel estimation for $60 \mathrm{GHz}$ wireless local area networks with massive receiving antennas," International Workshop on High Mobility Wireless Communications. IEEE, 2015:63-67. 